\title{
Comparison of long-term radial artery occlusion following trans-radial coronary intervention using 6-french versus 7 -french sheaths
}

\author{
Yanming Fan ${ }^{1}$, Qingmin $\mathrm{Wei}^{1}$, Junna $\mathrm{Cai}^{1}$, Yanbo Wang ${ }^{2}$, Xianghua $\mathrm{Fu}^{2}$ \\ ${ }^{1}$ Department of Cardiology, Xingtai People's Hospital, Xingtai, Hebei, China \\ ${ }^{2}$ Department of Cardiology, The Second Hospital of Hebei Medical University, Shijiazhuang, Hebei, China
}

\begin{abstract}
Background: The aim of this study was to explore the impact of 6-Fr and 7-Fr sheaths on the incidence of long-term radial artery occlusion (RAO) after trans-radial coronary intervention (TRI).

Methods: From September 2013 to January 2016, patients with ischemic heart disease including acute myocardial infarction and true bifurcation lesions were randomly assigned to 6-Fr group and 7-Fr group immediately after coronary angiography in a 1:1 ratio. The radial artery diameters were observed by ultrasound examination one day prior to TRI as well as at 30 days and 1 year after TRI. The primary endpoint was the incidence of RAO at 1-year after TRI. The secondary endpoints were the incidence of local vascular complications during hospitalization and changes of radial artery diameters within 1-year after TRI between the two groups. Additionally, multivariate logistic regression analysis was used to explore potential factors related to the incidence of long-term RAO after TRI.

Results: A total of 214 patients were enrolled and randomly assigned to 6-Fr group $(n=105)$ or 7 -Fr group $(n=109)$. There was no significant difference in the incidence of RAO at 1-year after TRI (8.57\% vs. $12.84 \%, p=0.313)$. Moreover, no significant difference was observed in the incidence of local vascular complications during hospitalization (20\% vs. $24.77 \%, p=0.403)$. After 1-year follow-up, no significant difference was found in radial artery diameters $(2.63 \pm 0.31 \mathrm{~mm} v \mathrm{~s} .2 .64 \pm 0.27 \mathrm{~mm}$, $p=0.802)$. Multivariate logistic analysis revealed that repeated TRI was an independent risk factor of long-term RAO 1 year after TRI $(O R=10.316,95 \%$ CI 2.928-36.351, $p=0.001)$.
\end{abstract}

Conclusions: Compared to 6-Fr sheath, 7-Fr sheath did not increase short-term or long-term incidence of RAO after TRI. (Cardiol J 2021; 28, 1: 49-57)

Key words: radial artery occlusion, trans-radial coronary intervention, radial artery diameter, artery sheath, local vascular complication

\section{Introduction}

With the rapid development of percutaneous coronary intervention (PCI) technique and persistent improvement of dedicated devices, trans-radial coronary intervention (TRI) has drastically advanced over the past two decades $[1,2]$. Compared to conventional femoral artery access, TRI has significantly reduced the incidence of local vascular complications. More importantly, it has brought overall therapeutic benefits with lower mortality and fewer major adverse cardiac events (MACE) rates [3, 4]. Nowadays, the vast majority of patients undergoing TRI procedure, 6 french (6-Fr) sheaths and guiding catheters are recommended as the first choice [5]. Usually, it is possible to do most of PCI procedures in regular cases through 6 -Fr guiding catheters. However,

Address for correspondence: Xianghua Fu, MD, PhD, FACC, FESC, Department of Cardiology, The Second Hospital of Hebei Medical University, No. 215 Heping West Road, Shijiazhuang 050000, Hebei Province, China, tel/fax: + 86 031166003803, e-mail: fuxh999@163.com

Received: 12.04.2019 Accepted: 20.08.2019

This article is available in open access under Creative Common Attribution-Non-Commercial-No Derivatives 4.0 International (CC BY-NC-ND 4.0) license, allowing to download articles and share them with others as long as they credit the authors and the publisher, but without permission to change them in any way or use them commercially. 
sometimes it is difficult to conduct complex coronary procedures through 6 -Fr guiding catheters, such as unprotected left main lesions, true bifurcation lesions treated with a two-stent strategy, severe calcified lesions requiring rotational atherectomy, and chronic total occlusion lesions requiring multiple wires, balloons and specialized devices (e.g., microcatheter, child-mother catheter, $1.75-\mathrm{mm}$ or larger burrs) simultaneously in one guiding catheter. Thus, a large-bore sheath (7-Fr) may be required to allow stronger back-up support and better materials delivery with no impact on hemodynamics monitoring and quality of coronary angiography, making the procedure easier and perhaps better [6]. Therefore, 7-Fr sheath and guiding catheters may be the better choice for complex coronary lesions as mentioned above.

Radial artery occlusion (RAO) is the most common local vascular complication, with a reported incidence of between $0.8 \%$ and $30 \%[7,8]$. A previous study showed that a dis-match between radial artery inner diameter and sheath outer diameter was an independent risk factor for RAO after TRI [5]. In contrast, a previous study revealed that 7-Fr sheath did not increase the incidence of RAO at 30-day follow-up after TRI in comparison to 6-Fr sheath [9]. Furthermore, few studies have focused on the impact of 7-Fr sheath on long-term RAO after TRI with inconsistent conclusions [10-12]. Thus, the aim of this study was to explore the impact of 6 -Fr and 7-Fr sheaths on the incidence of long-term RAO after TRI via vascular ultrasound.

\section{Methods}

\section{Patient population and study design}

This study was a prospective, randomized, controlled trial. From September 2013 to January 2016, patients with angina pectoris or evidence of myocardial ischemia and true bifurcation lesions confirmed by coronary angiography (CAG) in the Cardiology Department of the Second Hospital of Hebei Medical University were enrolled in this study. The true bifurcation lesions were defined as the diameter of side branch of more than $2 \mathrm{~mm}$ as well as degree of side branch ostium stenosis beyond $75 \%$ [9]. All coronary lesions were suitable for PCI treatment in this study. The exclusion criteria were: a negative Allen test, active inflammation, crossed over to other approaches (trans-femoral or trans-ulnar), repeated CAG or PCI via radial artery observed during the follow-up period, allergy to contrast agent, refusal to participate in the study, and inability to follow the protocol. The study protocol was approved by the ethics committee of the Second Hospital of Hebei Medical University (the IRB No. 2013L-22). Informed consent was obtained from each participant before TRI procedure. The study was conducted in accordance with the Declaration of Helsinki.

The enrolled patients were randomly assigned by computer-generated random numbers to either 6-Fr group or 7-Fr group immediately after CAG in a $1: 1$ ratio. Patients in 6 -Fr group underwent PCI with 6-Fr sheaths (outer diameter: $2.52 \mathrm{~mm}$, Radifocus, Terumo, Japan), while patients in 7-Fr group underwent PCI with 7-Fr sheaths (outer diameter: $2.85 \mathrm{~mm}$, Medtronic, USA). Patients could cross-over to the other group if necessary. Before inserting the 7-Fr sheath, sufficient local subcutaneous anesthesia with lidocaine and intraarterial nitroglycerin were administrated to avoid radial artery spasm.

\section{Trans-radial catheterization}

Coronary procedures were performed according to the standard technique of radial artery approach. The TRI was performed by the same experienced cardiac interventional team at a same center in both groups. The forearm was positioned beside the patient's body and the wrist was hyperextended. After local subcutaneous anesthesia with $1 \%$ lidocaine, radial artery puncture was carried out using a 20 -gauge needle (Terumo $\mathrm{Co}$ ) using the Seldinger technique and a 0.025-in straight tip guidewire (Terumo Co) was inserted through the needle. After removing the needle, a $16-\mathrm{cm} 6-\mathrm{Fr}$ hydrophilic sheath (Terumo Co) was placed over the guidewire. Subsequently, a bolus of unfractionated heparin (3000 IU) and $200 \mu \mathrm{g}$ nitroglycerin was administered through the sheath. The CAG was performed with 4-Fr Judkins diagnostic catheters (Terumo Co) or 5-Fr TIG diagnostic catheters (Terumo $\mathrm{Co}$ ). Moreover, weight-adjusted unfractionated heparin (70-100 IU/kg) was administered to maintain activated clotting time between 250 and $300 \mathrm{~s}$ during the PCI procedure. After TRI procedures, the radial arterial sheath was immediately pulled out and hemostasis (TR Band; Terumo Co) was achieved by radial compression. The TR Band was applied by inflating 13 to $15 \mathrm{~mL}$ of air at the puncture site. After each subsequent hour, the TR Band was gradually deflated $(2-3 \mathrm{~mL})$ until being completely removed. If there was bleeding during the deflation process, $2 \mathrm{~mL}$ of air would be injected to stop the bleeding and then was rechecked after $15 \mathrm{~min}$. 
Procedural variables among the two groups such as radial artery diameter (RAD) to sheath size ratio (A/S ratio), number of punctures (a needle pushed even just inside the skin was counted as a single attempt, regardless of skin puncture times), number of catheters used, heparin dose, procedure time, compression time, forearm hematomas and volume of contrast media were observed and evaluated.

\section{Ultrasound examination}

An experienced vascular sonographer blinded to the patients performed ultrasound examinations using an ultrasound system (Terason T3000, the USA) with a 5.0 to $12.0 \mathrm{MHz}$ linear transducer. Ultrasound-Doppler assessment of bilateral RAD, was conducted 1-day before the procedure at point 3 to $5 \mathrm{~cm}$ proximal to the styloid process of the radius bone. In addition, the RAD was assessed in 30 days and 1-year post TRI procedure. Moreover, the incidence of RAO in 1 year after TRI was observed, and was considered as the absence of antegrade flow in the radial artery observed by ultrasound.

\section{Study endpoints}

The primary endpoint of study was the incidence of RAO at 1 year after TRI between the two groups. On the other hand, the secondary endpoints of the study were the incidence of local vascular complications during hospitalization and RAD changes within 1 year after TRI in comparison to the baseline value before TRI between the two groups. The local vascular access-site complications included radial artery spasm (RAS), pseudoaneurysm, local hematoma, arteriovenous fistula and hand ischemia. The RAS was defined as a severe local pain and discomfort during catheter movement compelling the operator to stop the procedure and was confirmed by radial artery angiography. Local forearm hematomas were graded using the EASY classification [13].

\section{Statistical analysis}

Based on earlier studies it was speculated that at 1-year, the incidence of RAO after TRI procedure would be $6 \%$ in 6 -Fr group and $19 \%$ in $7-\mathrm{Fr}$ group [7]. Accordingly, at least 98 patients in each group were needed for a test power set at 0.8 and statistical level (2-sided) at 0.05 . Based on a $15 \%$ 1-year loss rate, at least 112 patients were needed in each group. All calculations were analyzed with SPSS statistical software (version 17.0; SPSS Inc, Chicago, Illinois). The continuous variables were expressed as means \pm standard deviation for normally distributed variables, while as median with interquartile range for non-normally distributed variables. The categorical variables were presented as percentages. Continuous variables were compared using the Student $t$ test for normally distributed values and the Mann-Whitney $\mathrm{U}$ test for non-normally distributed values. For proportions, if the expected frequency was $<5$, they were compared using the $\chi^{2}$ test or Fisher exact test. Multivariate logistic regression analysis was used to explore the possible factors associated with the incidence of long-term RAO after TRI. A 2 -sided $\mathrm{p}<0.05$ was considered statistically significant.

\section{Results}

\section{Baseline characteristics of the patients}

A total of 248 patients were enrolled and randomly divided into 6 -Fr group $(\mathrm{n}=124)$ and 7 -Fr group $(\mathrm{n}=124)$. During PCI procedure, 4 patients in the 6-Fr group were crossed-over to the 7-Fr group owing to their clinical need. All 7-Fr sheaths were successfully inserted. During follow-up, 34 (13.7\%) patients were excluded in this study, 15 patients in 6 -Fr group (8 patients experienced repeated TRI, 5 patients were lost to follow-up and 2 patients died), and 19 patients in 7-Fr group (10 patients experienced repeated TRI, 7 patients were lost to follow-up and 2 patients died). Finally, a number of 214 patients were enrolled and divided into the 6-Fr group $(\mathrm{n}=105)$ and the 7 -Fr group $(n=109)$ (Fig. 1). There were no significant differences in terms of age, gender, body mass index, risk factors of coronary artery disease, clinical presentation, previous TRI history or follow-up medication between the groups. The baseline clinical characteristics are shown in Table 1.

\section{Procedural characteristics of the patients}

No significant differences were found with respect to access artery, the number of puncture attempts, heparin dose, number of catheters used, volume of contrast medium or duration of compression. The ratio of radial artery inner diameter and sheath outer diameter in 6-Fr group was much higher than that in 7 -Fr group $(1.09 \pm 0.11$ vs. $0.96 \pm 0.13, \mathrm{p}<0.001)$. Besides, the procedural time of 6 -Fr group was much longer than that of $7-\mathrm{Fr}$ group $(74.27 \pm 12.58 \mathrm{~min}$ vs. $66.67 \pm 14.72 \mathrm{~min}$, $\mathrm{p}<0.001)$. The procedural characteristics are shown in Table 2. 


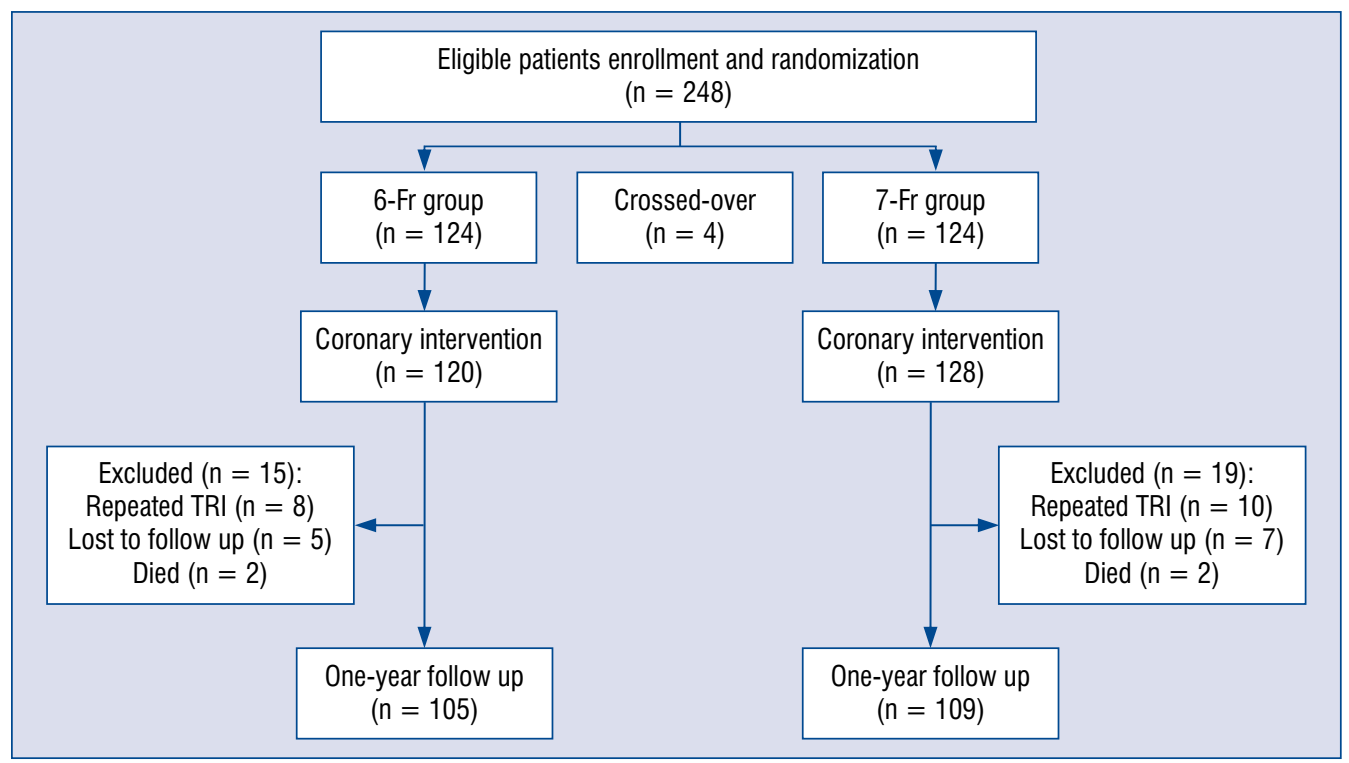

Figure 1. Flow chart of this study; TRI — trans-radial coronary intervention.

Table 1. Baseline clinical characteristics between the two groups.

\begin{tabular}{lccc}
\hline Variables & 6-Fr group $(\mathbf{n}=\mathbf{1 0 5})$ & 7-Fr group $(\mathbf{n}=\mathbf{1 0 9})$ & $\mathbf{P}$ \\
\hline Age $[$ years] & $58.08 \pm 10.07$ & $59.39 \pm 9.31$ & 0.325 \\
Male & $75(71.43 \%)$ & $82(75.22 \%)$ & 0.529 \\
BMI $\left[\mathrm{kg} / \mathrm{m}^{2}\right]$ & $25.39 \pm 3.13$ & $25.59 \pm 2.42$ & 0.587 \\
Hypertension & $54(51.43 \%)$ & $59(54.13 \%)$ & 0.692 \\
Diabetes & $35(33.33 \%)$ & $33(30.28 \%)$ & 0.631 \\
Hyperlipidemia & $41(39.05 \%)$ & $43(39.44 \%)$ & 0.952 \\
Current smoking & $36(34.28 \%)$ & $40(36.69 \%)$ & 0.712 \\
Clinical presentation: & & & \\
Stable angina & $4(3.81 \%)$ & $3(2.75 \%)$ & 0.664 \\
Unstable angina & $72(68.57 \%)$ & $74(67.89 \%)$ & 0.968 \\
NSTEMI & $23(21.90 \%)$ & $27(24.77 \%)$ & 0.738 \\
STEMI & $6(5.72 \%)$ & $5(4.59 \%)$ & 0.948 \\
Previous TRI history & $29(27.62 \%)$ & $33(30.28 \%)$ & 0.668 \\
Follow-up medication: & $105(100 \%)$ & & \\
ASA & $78(74.28 \%)$ & $109(100 \%)$ & $\mathrm{NS}$ \\
Clopidogrel & $27(25.72 \%)$ & $80(73.39 \%)$ & 0.882 \\
Ticagrelor & $102(97.14 \%)$ & $29(26.61 \%)$ & 0.882 \\
Statins & $45(42.86 \%)$ & $107(98.17 \%)$ & 0.621 \\
ACEI/ARB & $72(68.57 \%)$ & $47(43.12 \%)$ & 0.969 \\
Beta-blocker & $77(70.64 \%)$ & 0.742 \\
\hline
\end{tabular}

ASA - acetylsalicylic acid; ACEI — angiotensin-converting enzyme inhibitor; ARB — angiotensin receptor blocker; BMI — body mass index; NS - not significant; STEMI - ST-segment elevation myocardial infarction; NSTEMI — non-ST-segment elevation myocardial infarction;

$\mathrm{TRI}$ - trans-radial coronary intervention

\section{Incidence of RAO at 1 year after TRI}

Overall, RAO occurred in $23(10.75 \%)$ of the 214 patients (9 patients in 6-Fr group and 14 patients in 7-Fr group). Besides, all patients were asymptomatic and there was no incidence of acute hand ischemia. There was no significant difference of incidence of RAO in 1 year after TRI between the two groups ( $8.57 \%$ vs. $12.84 \%, \mathrm{p}=0.313$ ) (Fig. 2 ). 
Table 2. Procedural characteristics between the two groups.

\begin{tabular}{|c|c|c|c|}
\hline Variables & $6-F r$ group $(n=105)$ & 7-Fr group $(n=109)$ & $\mathbf{P}$ \\
\hline Access artery: & & & 0.353 \\
\hline Right radial artery & $97(92.38 \%)$ & $104(95.41 \%)$ & \\
\hline Left radial artery & $8(7.62 \%)$ & $5(4.59 \%)$ & \\
\hline $\mathrm{A} / \mathrm{S}$ ratio & $1.09 \pm 0.11$ & $0.96 \pm 0.13$ & $<0.001$ \\
\hline Numbers of puncture: & $1.12 \pm 0.47$ & $1.11 \pm 0.37$ & 0.813 \\
\hline Single puncture & $97(92.38 \%)$ & $99(90.82 \%)$ & 0.479 \\
\hline Anticoagulation drug: & & & 0.658 \\
\hline Heparin & $98(93.33 \%)$ & $100(91.74 \%)$ & \\
\hline Bivalirudin & $7(6.67 \%)$ & $9(8.26 \%)$ & \\
\hline Heparin dose [IU] & $10409.52 \pm 1836.83$ & $10389.91 \pm 1475.66$ & 0.931 \\
\hline Procedure time [min] & $74.27 \pm 12.58$ & $66.67 \pm 14.72$ & $<0.001$ \\
\hline Number of catheters & $2.10 \pm 0.33$ & $2.08 \pm 0.30$ & 0.616 \\
\hline Compression time $[\mathrm{h}]$ & $6.93 \pm 1.48$ & $6.81 \pm 1.42$ & 0.526 \\
\hline Use of GPI & $18(17.14 \%)$ & $22(20.18 \%)$ & 0.196 \\
\hline Volume of CM [mL] & $162.24 \pm 24.31$ & $159.32 \pm 24.77$ & 0.385 \\
\hline
\end{tabular}

A/S — radial artery inner diameter/sheath outer diameter; CM — contrast medium; GPI — platelet glycoprotein Ilb/Illa inhibitor

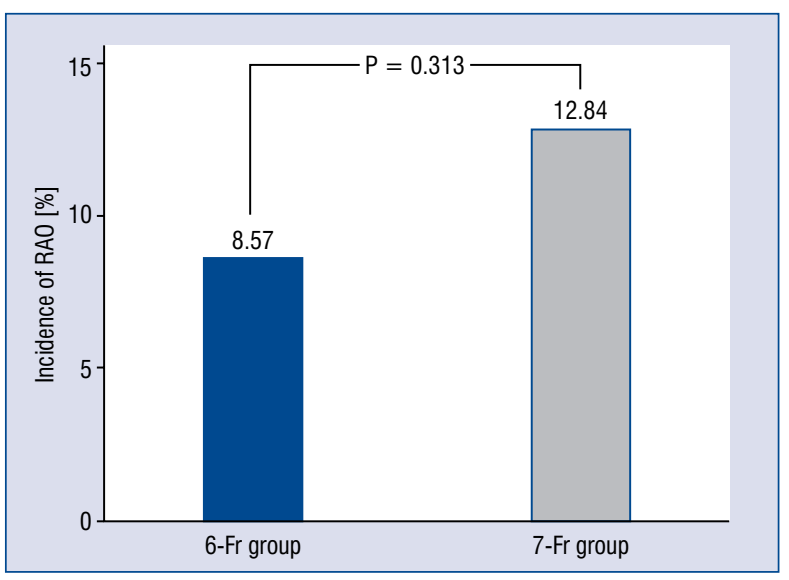

Figure 2. Incidence of radial artery occlusion (RAO) at 1 year after trans-radial coronary intervention.

Peri-procedure local vascular complications

There was no significant difference observed for the incidence of local vascular complications during hospitalization between the two groups ( $20 \%$ vs. $24.77 \%, p=0.403$ ). Moreover, there was no difference in RAO during hospitalization between the 6 - Fr (5.71\%) and 7-Fr groups $(7.34 \%, \mathrm{p}=0.613)$. Additionally, no obvious difference was found in the incidence of RAS, pseudoaneurysm, local hematoma, arteriovenous fistula and hand ischemia between the two groups (Table 3 ).

\section{Change of RADs within 1-year after TRI}

There was no significant difference of RADs at baseline between the two groups $(2.74 \pm 0.28 \mathrm{~mm}$ vs. $2.73 \pm 0.39 \mathrm{~mm}, \mathrm{p}=0.830$ ) and 30-day followup ( $2.69 \pm 0.39$ vs. $2.73 \pm 0.29 \mathrm{~mm}, \mathrm{p}=0.396)$. At 1-year follow-up, RADs in both the 6-Fr and 7-Fr groups were significantly reduced compared with baseline values before TRI (6-Fr group: $2.64 \pm 0.27$ $\mathrm{mm}$ vs. $2.74 \pm 0.28 \mathrm{~mm}, \mathrm{p}=0.009$; 7 -Fr group: $2.63 \pm 0.31 \mathrm{~mm}$ vs. $2.73 \pm 0.39 \mathrm{~mm}, \mathrm{p}=0.031$ ). However, no significant difference in RADs was found between the two groups at 1-year follow-up $(2.63 \pm 0.31 \mathrm{~mm}$ vs. $2.64 \pm 0.27 \mathrm{~mm}, \mathrm{p}=0.802)$. The change of RADs within 1-year after TRI are listed in Table 4 and Figure 3.

\section{Results of multiple logistic regression analysis}

Multivariate logistic analysis revealed that repeated TRI was an independent risk factor of longterm RAO in 1-year post TRI (odds ratio [OR] = $=10.316,95 \%$ confidence interval [CI] 2.928-36.351, $\mathrm{p}=0.001)$. However, RAD, the ratio of radial artery inner diameter/sheath and outer diameter, and the sheath size of the artery were not independent predictors of long-term RAO after TRI (Table 5).

\section{Discussion}

In this study, it was revealed that 7-Fr sheath did not increase the long-term incidence of RAO 
Table 3. Peri-procedure local vascular complications between two groups.

\begin{tabular}{lccc}
\hline Variables & 6-Fr group $(\mathbf{n}=\mathbf{1 0 5})$ & 7-Fr group (n = 109) & P \\
\hline Total number of complications & $21(20.00 \%)$ & $27(24.77 \%)$ & 0.403 \\
Radial artery occlusion & $6(5.71 \%)$ & $8(7.34 \%)$ & 0.631 \\
Radial artery spasm & $10(9.52 \%)$ & $12(11.01 \%)$ & 0.721 \\
Forearm hematoma: & $9(8.56 \%)$ & $13(11.93 \%)$ & 0.419 \\
$\leq$ II type & $8(7.61 \%)$ & $11(10.09 \%)$ & 0.525 \\
$>$ II type & $1(0.95 \%)$ & $2(1.83 \%)$ & 0.583 \\
Pseudoaneurysm & $1(0.95 \%)$ & $0(0 \%)$ & NS \\
Arteriovenous fistula & $0(0 \%)$ & $0(0 \%)$ & NS \\
Hand ischemia & $0(0 \%)$ & $0(0 \%)$ & NS \\
\hline
\end{tabular}

Table 4. Change of radial artery diameters between two groups.

\begin{tabular}{lccc}
\hline Variables & 6-Fr group $(\mathbf{n}=\mathbf{1 0 5})$ & 7-Fr group $(\mathbf{n}=109)$ & $\mathbf{P}$ \\
\hline Baseline $[\mathrm{mm}]$ & $2.74 \pm 0.28$ & $2.73 \pm 0.39$ & 0.830 \\
30 days after TRI [mm] & $2.73 \pm 0.29$ & $2.69 \pm 0.39$ & 0.396 \\
1 year after TRI [mm] & $2.64 \pm 0.27^{*}$ & $2.63 \pm 0.31^{*}$ & 0.802 \\
\hline
\end{tabular}

${ }^{*}$ Compared with baseline, $\mathrm{p}<0.05 ; \mathrm{TRI}$ - trans-radial coronary intervention

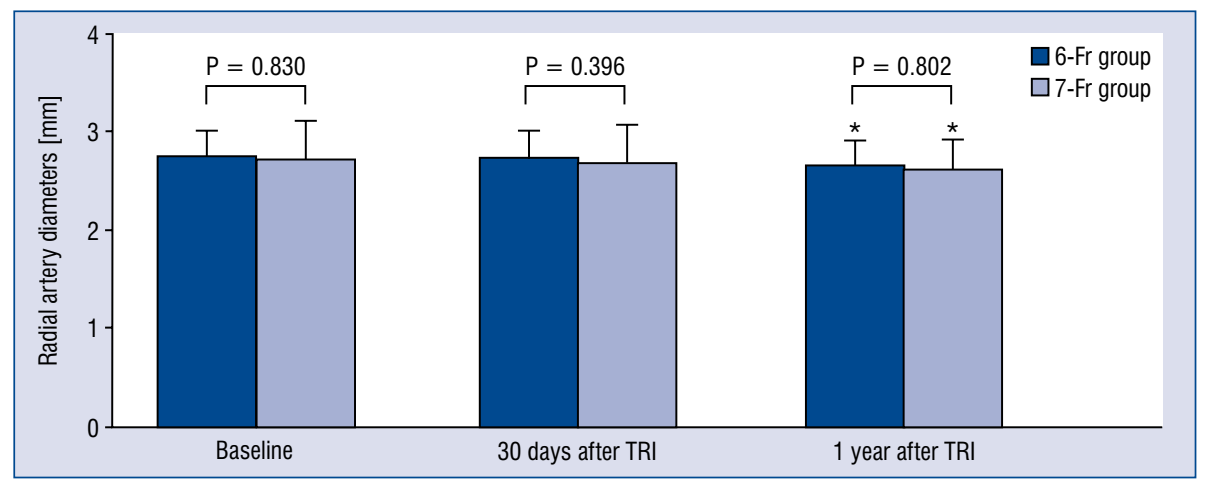

Figure 3. Change of radial artery diameters within 1-year after trans-radial coronary intervention (TRI); ${ }^{*}$ Compared with the baseline value, radial diameters were significantly decreased in both groups, $\mathrm{p}<0.05$.

after TRI compared to the 6-Fr sheath. Additionally, no apparent difference was observed on the RAD between 6 -Fr and 7-Fr sheaths in 1 year after TRI, but both of them were significantly reduced compared to the baseline value before TRI. In addition, repeated TRI was an independent risk factor of long-term RAO after TRI but not the RAD, A/S ratio or artery sheath size.

The use of the radial instead of the femoral approach is beneficial not only due to the reduction in the incidence of access-site complications, but also its capacity to reduce mortality and the risk of car- diac complications, especially in high-risk patients [14-16]. Moreover, the radial approach is also associated with more comfort for the patient, shorter hospitalization stays, and lower costs of treatment. However, RAO is one of the major complications of procedures performed via the radial artery [17, 18]. Although with an asymptomatic course, as well as the incidence of hand ischemia caused by RAO is extremely rare, RAO eliminates the ability to use the radial artery as an access for PCI in the future, to use it as a bypass conduit for patients undergoing coronary artery bypass surgery, or to 
Table 5. Predictors of radial artery occlusion by multivariate logistic analysis.

\begin{tabular}{lccc}
\hline Variables & \multicolumn{2}{c}{ Radial artery occlusion } & P \\
\cline { 2 - 3 } & Odds ratio & $95 \% \mathrm{Cl}$ & 0.930 \\
\hline Diabetes & 0.945 & $0.269-3.317$ & 0.001 \\
History of TRI & 10.316 & $2.928-36.351$ & 0.258 \\
Compression time & 0.790 & $0.526-1.188$ & 0.239 \\
Baseline radial artery diameter & 1.964 & $0.218-8.413$ & 0.393 \\
Radial artery spasm & 1.871 & $0.444-7.891$ & 0.624 \\
A/S $<1$ & 0.561 & $0.056-5.640$ & 0.914 \\
Artery sheath size & 0.933 & $0.263-3.309$ & 0 \\
\hline
\end{tabular}

$\mathrm{A} / \mathrm{S}$ - radial artery inner diameter/sheath outer diameter; $\mathrm{Cl}$ — confidence interval; TRI — trans-radial coronary intervention

use it for dialysis fistula. Previous studies have disclosed that RAO may be associated with the following factors such as female gender, diabetes, history of TRI, low dose of unfractionated heparin, longer hemostasis times, smaller radial artery and $\mathrm{A} / \mathrm{S}$ ratio $<1$ [7]. However, these findings have not been consistent among studies [19-22].

Traditionally, complex PCI has been performed at the femoral but not the radial approach due to the need for large size catheters. The use of 7-Fr sheath is limited mainly via radial artery owing to a risk of RAO after TRI. In a Japanese study using ultrasound evaluation, the $\mathrm{A} / \mathrm{S}$ ratio $<1$ predicted lower blood flow in radial artery after TRI procedure, but the impact on RAO has not been evaluated [5]. Besides, Uhlemann et al. [23] found that among 455 patients randomized to 5 -Fr or 6 -Fr sheaths, the 6 -Fr sheaths were associated with a significant higher incidence in RAO ( $13.7 \%$ vs. $30.5 \%$, p < $<0.001$ ). A previous study however, showed that 7-Fr sheath did not increase the incidence of RAO at 30 days follow-up after TRI compared with 6-Fr sheath ( $2 \%$ vs. $3.9 \%, p=0.70)$. In addition, in a single center registry [11], 175 patients were subjected to TRI via radial artery through a 7-Fr Radifocus ${ }^{\circledR}$ Introducer II (Terumo Corporation, Tokyo, Japan) with a 6-month follow-up, which observed an RAO rate of $6 \%(95 \%$ CI $3-11 \%)$ at manual assessment and $7 \%$ (95\% CI $4-12 \%)$ at Doppler evaluation, consistent to those reported in previous studies (ranging from 5\% to 38\%) using a 6-Fr sheath.

Nevertheless, all studies mentioned above were not randomized with a short-term follow-up, hence a bias in their selection may have occurred. Therefore, this prospective, randomized, controlled trial was conducted, and showed that 7-Fr sheath did not increase the long-term incidence of RAO after TRI compared with the 6-Fr sheath.
The reasons why 7-Fr sheath did not increase the long-term incidence of RAO after TRI may be associated with the following factors. Firstly, as a relatively high-volume TRI center, the operators have a rich experience of using 7 -Fr sheath via radial artery access. The high success rate of single puncture (97-99\%) may, to some extent, reduce injury to the radial artery. Moreover, the diameter of the radial artery, as measured by vascular ultrasound, is not a constant but a variable parameter. The diameter of radial artery can be reduced by stimuli and increased by intra-arterial administration of vasodilatory drugs such as nitroglycerin and verapamil. In this study, before insertion of $7-\mathrm{Fr}$ sheath, sufficient local subcutaneous anesthesia with lidocaine and intra-arterial nitroglycerin were administrated to decrease RAS and increase the compatibility between radial artery and sheath with a lower vascular resistance. Finally, patent hemostasis and adequate anticoagulation have also played important roles in preventing RAO.

Another interesting finding was that the RAD at 1-year follow-up after TRI was significantly lower than baseline value before TRI in both of 6-Fr and 7-Fr groups, however, no obvious difference was observed between the two groups. Previous studies documented that TRI was related to intimal hyperplasia in the cannulated radial artery, as revealed by vascular imaging modalities such as intravascular ultrasound and optical coherence tomography [24-26]. In addition, recent studies have shown that the TRI procedure may lead to impaired flow-mediated dilation during long-term follow-up, which has been widely used for the non-invasive assessment of endothelium-dependent vasodilation response [27-29]. Therefore, it was assumed that the reduction of RAD may be associated with structural damage and impaired endothelial func- 
tion of the cannulated radial artery due to chronic inflammatory and the proliferative process. This could explain why repeated TRI was an independent risk factor of long-term RAO at one year after TRI in the present study.

The current study has several potential limitations. First, it is a relatively small-scale study, conducted in a single center. In addition, optical coherence tomography could have provided more details about structural damage such as intimal tears and medial dissections together with chronic intimal modifications but was not used.

\section{Conclusions}

In conclusion, this study showed that $7-\mathrm{Fr}$ sheath did not increase the long-term incidence of RAO after TRI compared with 6 -Fr sheath. Therefore, 7-Fr sheath in the radial artery access could be feasible and safe for complex coronary lesions, especially at experienced centers.

\section{Conflict of interest: None declared}

\section{References}

1. Mason PJ, Shah B, Tamis-Holland JE, et al. An Update on Radial Artery Access and Best Practices for Transradial Coronary Angiography and Intervention in Acute Coronary Syndrome: A Scientific Statement From the American Heart Association. Circ Cardiovasc Interv. 2018; 11(9): e000035, doi: 10.1161/ HCV.0000000000000035, indexed in Pubmed: 30354598.

2. Rao SV, Cohen MG, Kandzari DE, et al. The transradial approach to percutaneous coronary intervention: historical perspective, current concepts, and future directions. J Am Coll Cardiol. 2010; 55(20): 2187-2195, doi: 10.1016/j.jacc.2010.01.039, indexed in Pubmed: 20466199.

3. Ferrante G, Rao SV, Jüni P, et al. Radial versus femoral access for coronary interventions across the entire spectrum of patients with coronary artery disease: a meta-analysis of randomized trials. JACC Cardiovasc Interv. 2016; 9(14): 1419-1434, doi: 10.1016/j.jcin.2016.04.014, indexed in Pubmed: 27372195.

4. Sandoval Y, Burke MN, Lobo AS, et al. Contemporary arterial access in the cardiac catheterization laboratory. JACC Cardiovasc Interv. 2017; 10(22): 2233-2241, doi: 10.1016/j.jcin.2017.08.058, indexed in Pubmed: 29169493.

5. Saito S, Ikei H, Hosokawa G, et al. Influence of the ratio between radial artery inner diameter and sheath outer diameter on radial artery flow after transradial coronary intervention. Catheter Cardiovasc Interv. 1999; 46(2): 173-178, doi: 10.1002/(SICI)1522726X(199902)46:2<173::AID-CCD12>3.0.CO;2-4, indexed in Pubmed: 10348538.

6. Juergens CP, Hallani H, Leung DYC, et al. Comparison of 6 and 7 French guiding catheters for percutaneous coronary intervention: results of a randomised trial with a vascular ultrasound endpoint. Catheter Cardiovasc Interv. 2005; 66(4): 528-534, doi: 10.1002/ccd.20534, indexed in Pubmed: 16208714.
7. Rashid M, Kwok CS, Pancholy S, et al. Radial artery occlusion after transradial interventions: a systematic review and meta-analysis. J Am Heart Assoc. 2016; 5(1), doi: 10.1161/ JAHA.115.002686, indexed in Pubmed: 26811162.

8. Wagener JF, Rao SV. Radial artery occlusion after transradial approach to cardiac catheterization. Curr Atheroscler Rep. 2015; 17(3): 489, doi: 10.1007/s11883-015-0489-6, indexed in Pubmed: 25651786.

9. Fu XH, Geng W, Gu XH, et al. Feasibity and safety of using 7 Fr guiding catheter via transradial artery approach for coronary bifurcation lesions undergoing preliminary administration of vasodilators (in Chinese). Chin J Intervent Cardiol. 2014; 22(12): 749-754.

10. Aminian A, Iglesias JF, Van Mieghem C, et al. First prospective multicenter experience with the 7 French Glidesheath slender for complex transradial coronary interventions. Catheter Cardiovasc Interv. 2017; 89(6): 1014-1020, doi: 10.1002/ccd.26773, indexed in Pubmed: 27567021.

11. Tumscitz C, Pirani L, Tebaldi M, et al. Seven french radial artery access for PCI: a prospective single-center experience. Int J Cardiol. 2014; 176(3): 1074-1075, doi: 10.1016/j.ijcard.2014.07.134, indexed in Pubmed: 25127334.

12. Levin D, Adawi S, Halon DA, et al. Long-term radial artery patency following transradial coronary catheterization via a 7 -fr sheath. Isr Med Assoc J. 2016; 18(5): 290-293, indexed in Pubmed: 27430087.

13. Bertrand OF, De Larochellière R, Rodés-Cabau J, et al. A randomized study comparing same-day home discharge and abciximab bolus only to overnight hospitalization and abciximab bolus and infusion after transradial coronary stent implantation. Circulation. 2006; 114(24): 2636-2643, doi: 10.1161/CIRCULATIONAHA.106.638627, indexed in Pubmed: 17145988.

14. Rao SV, Tremmel JA, Gilchrist IC, et al. Best practices for transradial angiography and intervention: a consensus statement from the society for cardiovascular angiography and intervention's transradial working group. Catheter Cardiovasc Interv. 2014; 83(2): 228-236, doi: 10.1002/ccd.25209, indexed in Pubmed: 24123781.

15. Valgimigli M, Gagnor A, Calabró P, et al. Radial versus femoral access in patients with acute coronary syndromes undergoing invasive management: a randomised multicentre trial. Lancet. 2015; 385(9986): 2465-2476, doi: 10.1016/S0140-6736(15)60292-6, indexed in Pubmed: 25791214.

16. Jolly SS, Yusuf S, Cairns J, et al. Radial versus femoral access for coronary angiography and intervention in patients with acute coronary syndromes (RIVAL): a randomised, parallel group, multicentre trial. Lancet. 2011; 377(9775): 1409-1420, doi: 10.1016/ S0140-6736(11)60404-2, indexed in Pubmed: 21470671.

17. Hahalis G, Tsigkas G, Kakkos S, et al. Vascular complications following transradial and transulnar coronary angiography in 1600 consecutive patients. Angiology. 2016; 67(5): 438-443, doi: 10.1177/0003319715592095, indexed in Pubmed: 26124493.

18. Garg N, Madan BK, Khanna R, et al. Incidence and predictors of radial artery occlusion after transradial coronary angioplasty: Doppler-guided follow-up study. J Invasive Cardiol. 2015; 27(2): 106-112, indexed in Pubmed: 25661763.

19. Pancholy SB, Bertrand OF, Patel T. Comparison of a priori versus provisional heparin therapy on radial artery occlusion after transradial coronary angiography and patent hemostasis (from the PHARAOH Study). Am J Cardiol. 2012; 110(2): 173-176, doi: 10.1016/..amjcard.2012.03.007, indexed in Pubmed: 22497680. 
20. Edris A, Gordin J, Sallam T, et al. Facilitated patent haemostasis after transradial catheterisation to reduce radial artery occlusion. EuroIntervention. 2015; 11(7): 765-771, doi: 10.4244/EIJV1117A153, indexed in Pubmed: 26603985.

21. Pancholy SB, Bertrand OF, Patel T. Comparison of a priori versus provisional heparin therapy on radial artery occlusion after transradial coronary angiography and patent hemostasis (from the PHARAOH Study). Am J Cardiol. 2012; 110(2): 173-176, doi: 10.1016/j.amjcard.2012.03.007, indexed in Pubmed: 22497680.

22. Zhou Yj, Zhao Yx, Cao Z, et al. [Incidence and risk factors of acute radial artery occlusion following transradial percutaneous coronary intervention]. Zhonghua Yi Xue Za Zhi. 2007; 87(22): 1531-1534, indexed in Pubmed: 17785103.

23. Uhlemann M, Möbius-Winkler S, Mende M, et al. The Leipzig prospective vascular ultrasound registry in radial artery catheterization: impact of sheath size on vascular complications. JACC Cardiovasc Interv. 2012; 5(1): 36-43, doi: 10.1016/j. jcin.2011.08.011, indexed in Pubmed: 22230148.

24. Di Vito L, Burzotta F, Trani C, et al. Radial artery complications occurring after transradial coronary procedures using long hydrophilic-coated introducer sheath: a frequency domain-optical coherence tomography study. Int J Cardiovasc Imaging. 2014; 30(1): 21-29, doi: 10.1007/s10554-013-0284-9, indexed in Pubmed: 24154615 .
25. Wakeyama T, Ogawa H, Iwami T, et al. Intima-media thickening of the radial artery after transradial intervention. An intravascular ultrasound study. J Am Coll Cardiol. 2003; 41(7): 1109-1114, doi: 10.1016/s0735-1097(03)00089-5, indexed in Pubmed: 12679209.

26. Yonetsu T, Kakuta T, Lee T, et al. Assessment of acute injuries and chronic intimal thickening of the radial artery after transradial coronary intervention by optical coherence tomography. Eur Heart J. 2010; 31(13): 1608-1615, doi: 10.1093/eurheartj/ehq102, indexed in Pubmed: 20413398.

27. Nakata T, Ikeda S, Koga S, et al. Impact of catheter sheath insertion into the radial artery on vascular endothelial function assessed by reactive hyperemia peripheral arterial tonometry. Int Heart J. 2015; 56(5): 489-494, doi: 10.1536/ihj.15-094, indexed in Pubmed: 26370365.

28. Yan Z, Zhou Y, Zhao Y, et al. Impact of transradial coronary procedures on radial artery function. Angiology. 2014; 65(2): 104-107, doi: 10.1177/0003319713479650, indexed in Pubmed: 23460113.

29. Fan Y, Fu X, Wang Y, et al. Effect of long-term administration of nicorandil on endothelial function of the radial artery in patients with angina undergoing transradial percutaneous coronary intervention. Angiology. 2017; 68(7): 633-639, doi: 10.1177/0003319716675720, indexed in Pubmed: 27815334. 